\title{
Sensitivity of Intrinsic Permeability to Electrokinetic Coupling in Shaly and Clayey Porous Media
}

\author{
Alessandro Brovelli • Giorgio Cassiani
}

Received: 3 February 2009 / Accepted: 2 September 2009 / Published online: 2 October 2009

(C) Springer Science+Business Media B.V. 2009

\begin{abstract}
Classical Darcy's law assumes that the intrinsic permeability of porous media is only dependent on the micro-geometrical and structural properties of the inner geometry of the medium. There are, however, numerous experimental evidences that intrinsic permeability of shaly and clayey porous material is a function of the fluid phase used in the experiments. Several pore-scale processes have been proposed to explain the observed behavior. In this study, we conduct a detailed investigation of one such mechanism, namely the electrokinetic coupling. We have developed a numerical model to simulate this process at the pore-scale, incorporating a refined model of the electrical double layer. The model is used to conduct a detailed sensitivity analysis to elucidate the relative importance of several chemical-physical parameters on the intensity of the electrokinetic coupling. We found that permeability reduction due to this mechanism is likely to occur only if the effective pore-radius is smaller than $10^{-6} \mathrm{~m}$. We also observed that electrokinetic coupling is strongly sensitive to electrophoretic mobility, which is normally reduced in clays compared to free-water conditions. Based on these findings, we set up a suite of stochastic pore-network simulations to quantify the extent of permeability reduction. We found that only if the effective pore-radius is ranging from $5 \times 10^{-7} \mathrm{~m}$ to $5 \times 10^{-8}$, electrokinetic coupling can be responsible for a $5-20 \%$ reduction of the intrinsic permeability, and, therefore, this mechanism has a minor impact on situations of practical environmental or mining interest.
\end{abstract}

Keywords Intrinsic permeability - Electrical double layer · Electrokinetic coupling · Darcy's law $\cdot$ Sensitivity analysis

\footnotetext{
A. Brovelli $(\bowtie)$

Ecological Engineering Laboratory, Institut d'ingénierie de l'environnement, Ecole Polytechnique Fédérale de Lausanne, Batiment GR, Station 2, 1015 Lausanne, Switzerland e-mail: alessandro.brovelli@epfl.ch

G. Cassiani

Dipartimento di Geoscienze, Università di Padova, Via Giotto 1, 35127 Padova, Italy

e-mail: giorgio.cassiani@unipd.it
} 


\section{List of Symbols}

$e\left(=1.602 \times 10^{-19} \mathrm{C}\right)$

$\varepsilon_{0}\left(=8.854 \times 10^{-12} \mathrm{~F} \mathrm{~m}^{-1}\right)$

$\varepsilon_{\mathrm{r}}(=80)$

$k_{\mathrm{B}}\left(=1.381 \times 10^{-23} \mathrm{~J} \mathrm{~K}^{-1}\right)$

$N_{\mathrm{a}}\left(=6.022 \times 10^{23} \mathrm{~mol}^{-1}\right)$

$F\left(=9.648 \times 10^{4} \mathrm{C} \mathrm{mol}^{-1}\right)$

$\mu_{i}^{\text {conv }}\left(=5.0 \times 10^{-8} \mathrm{~m}^{-2} \mathrm{~V}^{-1} \mathrm{~s}^{-1}\right)$

$\kappa^{-1}(\mathrm{~m})$

$P(\mathrm{~Pa})$

$\phi(\mathrm{V})$

$\zeta(\mathrm{V})$

$\psi(\mathrm{V})$

$\varphi_{\mathrm{d}}(\mathrm{V})$

$C_{i}\left(\mathrm{~mol} \mathrm{1}^{-1}\right)$

$T(\mathrm{~K})$

$z_{i}$

$u\left(\mathrm{~m} \mathrm{~s}^{-1}\right)$

$\rho_{\mathrm{e}}\left(\mathrm{C} \mathrm{m}^{-3}\right)$

$a(\mathrm{~m})$

$l(\mathrm{~m})$

$\eta\left(=1 \times 10^{-3} \mathrm{~N} \mathrm{~s} \mathrm{~m}^{-2}\right)$

$\sigma\left(\mathrm{S} \mathrm{m}^{-1}\right)$

$\Sigma_{\mathrm{S}}\left(\mathrm{S} \mathrm{m}^{-2}\right)$

$Q\left(\mathrm{~m}^{3} \mathrm{~s}^{-1}\right)$

$I(\mathrm{~A})$

$k\left(\mathrm{~m}^{2}\right)$

$k_{0}\left(\mathrm{~m}^{2}\right)$

$\Gamma_{\mathrm{s}}^{0}\left(\mathrm{~nm}^{-2}\right)$

$K_{\mathrm{m}}\left(=1 \times 10^{-3}\right)$

$K_{\mathrm{Me}}\left(=1 \times 10^{-7.5}\right)$

$\alpha_{\sigma}\left(=2.30 \times 10^{-2} \mathrm{~K}^{-1}\right)$

$\alpha_{\Sigma}\left(=2.30 \times 10^{-2} \mathrm{~K}^{-1}\right)$

$\alpha \zeta\left(=1.71 \times 10^{-2} \mathrm{~K}^{-1}\right)$

$n(=0.30)$

$\rho_{\mathrm{g}}\left(=2600 \mathrm{~kg} \mathrm{~m}^{-3}\right)$
Elementary charge

Vacuum permittivity

Dielectric constant of water

Boltzmann's constant

Avogadro number

Faraday's constant

Conventional electrophoretic ion mobility, specie $i$

Debye length

Fluid pressure

Electrical potential

$\zeta$-potential

Electrical potential of the diffuse layer

Electrical potential of the Stern layer

Concentration, specie $i$

Temperature

Valence, specie $i$

Fluid velocity

Electrical charge density

Capillary radius

Capillary length

Water viscosity

Electrical conductivity

Surface conductance

Water flow rate

Electrical current

Intrinsic permeability (considering electrokinetic

coupling)

Intrinsic permeability (without electrokinetic coupling)

Surface charge density

Equilibrium constant, silossanic/silanoic groups

Sorption equilibrium constant, cations

Temperature correction coefficient, electrical conductivity

Temperature correction coefficient, surface conductance Temperature correction, $\zeta$-potential

Porosity

Grain (i.e., solid phase) density

\section{Introduction}

Single and multiphase flow in porous media is normally modeled using Darcy's law, with the fluid flow linearly dependent on the hydraulic gradient. The proportionality constant is the hydraulic conductivity, a function of the physical properties of both the fluid and the porous medium, $K=\mathrm{k} \rho \mathrm{g} / \eta$ where $\eta$ and $\rho$ are the viscosity and density of the fluid, respectively, $g$ is the acceleration of gravity, and $k$ the intrinsic permeability, a phenomenological 
parameter related to the resistance to flow of the porous medium. In the classical and widely accepted theory, intrinsic permeability is solely related to the micro-geometrical features of the pore-space, namely porosity, pore-connectivity and tortuosity (e.g., Bear 1988; Dullien 1992). Nevertheless, numerous laboratory experiments observed substantial deviations from this theory, since it is found that the apparent intrinsic permeability changes as a function of the mobile phase used to measure the intrinsic permeability. Most of these studies compare the measurements with water and with an inert gas as a mobile phase, for example nitrogen (Jones and Owens 1980; Dana and Skoczylas 1999; Baraka-Lokmane 2002). After corrections for the gas slippage (or Klinkenberg) effect (Guéguen and Palciauskas 1994), it is observed that for certain classes of porous materials the intrinsic permeability obtained with gas $\left(k_{g}\right)$ is systematically larger than the same parameter measured with water $\left(k_{w}\right)$. For example, Baraka-Lokmane 2002 analyzed a set of sandstone cores, and found that the gas permeability was 1-2 times larger than water permeability in $61 \%$ of the cores, while in the remainder, the difference was a factor in the range 2-5. Similar results were obtained by other authors (e.g., Jones and Owens 1980; Dana and Skoczylas 1999), and an even larger range of the $k_{g} / k_{w}$ ratio was obtained. Other studies instead observed changes in the intrinsic permeability as a function of the composition and salinity of the water phase used in the experiments (Raffenspergen and Ferrel 1991).

In order to describe the observations and estimate the permeability to water of porous materials from the measurements conducted using an inert gaseous phase, Swanson (1978, 1981) and Juhàsz (1979) developed some relationships linking $k_{g}$ and $k_{w}$. The proposed equations consider petrophysical parameters such as the total and effective porosity and the pore-size distribution as recovered from mercury intrusion experiments, but also have one or more adjustable empirical parameter to be calibrated on the laboratory measurements.

Several processes have been identified as being potentially responsible for the observed difference between air and water permeability. According to Baraka-Lokmane (2002) the reduced water permeability of shaly sandstones is the result of three synergetic processes, i.e., (i) migration of clays and fine particles clogging the pores, (ii) partial desaturation of the samples during the experiment, and/or incomplete core saturation prior to start the measures, and (iii) mechanical breakdown of the clay fabric, and subsequent reduction of the effective pore sizes. Other studies, however, have pointed out that an additional permeability decrease can result from a number of chemical-physical processes triggered by the presence of an electrically charged surface (e.g., Wilson 1982; Raffenspergen and Ferrel 1991; Soma and Papadopulos 1995; Lyklema 2001; Revil et al. 1999b; Revil and Pessel 2002 and references therein). The charged water-solid matrix interface has a twofold effect, (i) increases the viscosity of the water phase near the pore wall, and (ii) induces an electrokinetic coupling. Both mechanisms are related to the negative charges on the surface of the clays, which modify the transport properties of charged ions and water molecules at the microscopic level. In this article, following the conclusions of Revil and Pessel (2002), where it is found that the classical Darcy's equation does not fully apply to silty shales, we aim at analyzing the extent of permeability reduction due to the coupling between water flow and transport of ions in an electric field. All the other mechanisms that could possibly contribute are disregarded. We focus mainly on medium to fine grained materials such as shaly sandstones, shales and silty shales because most of the experimental results and evidences have been obtained using these materials. Shales and shaly sandstones are often found in or near oil and gas reservoirs, and, therefore, a process-based understanding of the mechanisms responsible for the permeability discrepancies is required to accurately forecast hydrocarbon production. Indeed, the same processes are also likely to reduce water flow in clayey porous media (see e.g., Keijzer et al. 1999 and the references therein). Fine-grained, shaly, 
and silty materials are commonly used to construct liners for waste containment in landfills, and to insulate nuclear waste repositories. Engineering design of such systems would certainly benefit from a better understanding of the processes affecting the permeability reduction.

The article is organized as follows. First, in Sect. 2, we briefly introduce the theory of the electrokinetic coupling, and we present the implementation of a numerical model capable of computing the intrinsic permeability of a pore-network. Within the same modeling framework, all the chemical-physical properties of the porous medium and pore fluid can be varied, and, therefore, we can investigate the resulting change in intrinsic permeability. To this end, in Sect. 3, we conduct a detailed sensitivity analysis to understand which are the main parameters controlling the response of our model, and to assess whether it is realistic or not to expect some permeability reduction as a result of the electrokinetic coupling. Finally, in Sect. 4, we perform a suite of Monte Carlo simulations on pore-networks to evaluate a possible relationship between pore-size distribution and the permeability decrease.

\section{Model Development}

\subsection{Electro-Kinetic Coupling}

In porous media with an electrically charged internal surface, the concentration of ions is not uniform within the cross section of a pore. Instead, ions tend to accumulate at the interface between the solid matrix and the pore-fluid to equilibrate the excess of charge of the surface. As a result, at steady state, with no external pressure gradient (i.e., no water flow) an electrical double layer (EDL) exists near the inner solid matrix and the macroscopic electrical potential is zero (electro-neutrality condition). In the presence of a pressure gradient, the pore-fluid moves, and, therefore, perturbs the ion distribution near the pore walls. As a result, electroneutrality disappears and an electrical potential is generated (named spontaneous potential, SP). Although this description of the processes is only simplified, it should highlight that electrokinetic coupling may result in an observable reduction of the intrinsic permeability. In fact, some energy is dissipated to move the charged ions within the electrical field near the solid matrix surface, with an overall behavior similar to an increase of shear stress in the vicinity of the pore wall. Clearly, this mechanism only exists when the pore fluid is water (or, in general, a polar solvent), because it requires the presence of dissociated, charged ions. Electro-kinetic processes have been intensively studied over the last two decades, and a complete review of the numerous phenomena connected can be found in the literature (e.g., Revil et al. 1999a; Revil et al. 1999b).

The mathematical description of the electrokinetic coupling is well known, and has been applied in several fields (e.g Hildreth 1970; Bowen and Jenner 1995; Li and Kwok 2003; Capuani et al. 2004, 2005; Hlushkou et al. 2006). For simplicity, in this study, we assume that the pore fluid contains a single, symmetric monovalent electrolyte. We also assume that the activity coefficients are constant and equal to 1 . Note, however, that the same mathematical description that we apply in this study can be used to describe the behavior of a more complex water solution.

At steady state, electrokinetic flow in a pore can be modeled using the Navier-Stokes equation, with an additional term linked to the electrostatic component ( $\mathrm{Li}$ and Kwok 2003; Capuani et al. 2004): 


$$
\eta \frac{1}{r} \frac{\mathrm{d}}{\mathrm{d} r}\left(r \frac{\mathrm{d} u}{\mathrm{~d} r}\right)-\nabla p-\rho_{\mathrm{e}} \nabla \phi=0
$$

where $\eta$ is the fluid viscosity, $r$ the radial coordinate, $u(r)$ the fluid velocity along the pore, $\nabla p$ the fluid pressure gradient and $\nabla \phi$ the gradient of electrical potential (parallel to the flow direction). The net charge density $\rho_{\mathrm{e}}$ can be computed using Poisson's equation:

$$
\rho_{\mathrm{e}}(r)=-\varepsilon_{r} \varepsilon_{0} \frac{1}{r} \frac{\mathrm{d}}{\mathrm{d} r}\left(r \frac{\mathrm{d} \psi}{\mathrm{d} r}\right)
$$

where $\varepsilon_{r}$ is the relative dielectric constant, $\varepsilon_{0}$ is the permittivity of vacuum, and $\psi(r)$ the electrical potential of the EDL near the charged pore-wall.

Analytical solutions for these coupled equations are available only for very simple geometries. Therefore, to accurately solve the electrokinetic flow problem in complex systems, the use of numerical tools (e.g., finite element or Lattice Boltzmann methods) is beneficial (Li and Kwok 2003; Capuani et al. 2004, 2005). Sometimes, however, the geometry of interest can be approximated with a combination of simpler elements, with a known analytical solution. With this approach, the electrical potential and pressure distribution are computed at the nodes of a network (Bernabé 1998; Xuan and Li 2004). The main advantage of this simplified approach is the significantly reduced computational load. On the other hand, with this method, an accurate representation of the real geometry may not be possible, and only some of the bulk properties of the original geometry can be retained. Indeed, numerous studies (Dullien 1992; Rajaram 1997; Blunt 1998; Peat et al. 2000; Vogel 2000) found that intrinsic permeability as well as several transport properties of porous media and other geo-materials can be effectively predicted using networks of pores with idealized geometry (for example, a cylindrical shape). Consequently, in this study, we adopted this simplified but practical approach. Details of the implementation are given in the next sub-sections.

\subsection{Analytical Solutions for Cylindrical Capillaries}

We consider a cylindrical capillary with radius $a$ and length $l$ (Fig. 1). Combining Eqs. 1 and 2 and integrating (boundary conditions are $u(a)=0, \psi(a)=\zeta$, and $u^{\prime}(0)=0, \psi^{\prime}(0)=0$, where the prime indicates the first derivative with respect to $r$ ) leads to an expression for the fluid velocity within the capillary (Bowen and Jenner 1995; Bernabé 1998; Xuan and Li 2004):

$$
u(r)=\frac{1}{4 \eta}\left(r^{2}-a^{2}\right) \nabla p-\frac{\varepsilon_{r} \varepsilon_{0} \zeta}{\eta}\left(\frac{\psi(r)}{\zeta}-1\right) \nabla \phi
$$

Owing to the electrokinetic coupling, the current density $j$ is the sum of two components, namely, (i) the convective transport of ions induced by the water flow and (ii) the ionic displacement as a result of the electric field (in our application, the electric field is created by the surface charge):

$$
j(r)=z e\left(n_{+}-n_{-}\right) u(r)-\mu^{\mathrm{conv}} z e\left(n_{+}+n_{-}\right) \nabla \phi
$$

where $n_{+}$and $n_{-}$are the volume densities of cations and anions, respectively.

At steady state, electrical charges follow a Boltzmann distribution:

$$
n_{ \pm}(r)=n_{0} \exp \left(\mp \frac{z e \psi(r)}{k_{\mathrm{B}} T}\right) .
$$




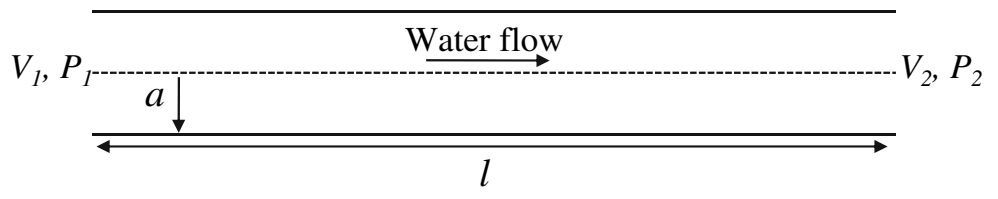

Fig. 1 Sketch of the capillary used in the model

Combining Eqs. 4 and 5 we obtain

$$
j(r)=\rho_{\mathrm{e}}(r) u(r)-\sigma \cosh \left(\frac{z e \psi(r)}{k_{\mathrm{B}} T}\right) \nabla \phi .
$$

The net charge density is defined as

$$
\rho_{\mathrm{e}}=-2 z e n_{o} \sinh \left(\frac{z e \psi}{k_{\mathrm{B}} T}\right) .
$$

Integrating Eqs. 3 and 6 over the cross section, the volumetric flow rate $Q$ and the electrical current $I$ in a capillary of length $l$ are recovered:

$$
\begin{aligned}
Q & =L_{11} \nabla p+L_{12} \nabla \phi \\
I & =L_{21} \nabla p+L_{22} \nabla \phi
\end{aligned}
$$

where $L_{i j}$ are the phenomenological coefficients, functions of both the capillary geometry and the chemical-physical properties of the pore-fluid and solid/water phase interface:

$$
\begin{aligned}
& L_{11}=-\frac{\pi a^{4}}{8 \eta l} \\
& L_{12}=L_{21}=\frac{\pi \varepsilon_{r} \varepsilon_{0} a^{2} \zeta}{\eta l} G_{1} \\
& L_{22}=-\frac{\pi \varepsilon_{r}^{2} \varepsilon_{0}^{2} \zeta^{2}}{\eta l} G_{2}-\frac{\pi a^{2} \sigma}{l} G_{3}
\end{aligned}
$$

For the Onsager reciprocity theorem, it has been shown that the cross-coupling coefficients $L_{12}$ and $L_{21}$ are equal (Onsager 1931; Bockris and Reddy 1998).

Using the Debye-Huckel linearization of the EDL, the constants $G_{1}, G_{2}, G_{3}$ can be computed (Bowen and Jenner 1995; Bernabé 1998; Xuan and Li 2004):

$$
\begin{aligned}
G_{1} & =1-\frac{2 I_{1}(\kappa a)}{\kappa a I_{0}(\kappa a)} \\
G_{2} & =(\kappa a)^{2}\left[\frac{I_{1}^{2}(\kappa a)}{I_{0}^{2}(\kappa a)}+\frac{2 I_{1}(\kappa a)}{\kappa a I_{0}(\kappa a)}-1\right] \\
G_{3} & =2 \int_{0}^{a}\left(\frac{r}{a}\right) \cosh \left(\frac{z e \zeta}{k_{\mathrm{B}} T} \frac{I_{0}(\kappa r)}{I_{0}(\kappa a)}\right) d\left(\frac{r}{a}\right)
\end{aligned}
$$

where $I_{0}($.$) and I_{1}($.$) are the zero- and first-order modified Bessel functions of the first kind,$ respectively.

The dimensionless parameter $\kappa a$ represents the size of the capillary $(a)$ relative to the thickness of the EDL $\left(\kappa^{-1}\right)$, and, therefore, it is a useful measure of the intensity of electrokinetic coupling. Equations 8-15 fully describe the electrokinetic coupling process in a cylindrical capillary. However, owing to the integral in Eq. 15, closed-form solutions are not 
available unless it is assumed $G_{3}=1$. This simplification is physically equivalent to neglecting the changes in surface conductance on the surface of the capillary, and is valid only when $\kappa a$ is large (i.e., the thickness of the EDL is small compared to the size of the capillary) (Xuan and Li 2004). This is clearly a situation in which the reduction of the porous medium permeability is negligible and is therefore not of interest in this study.

\subsection{Chemical-Physical Properties of Porous Media}

Owing to inherent complexities in both the experimental determination of the chemicalphysical properties of the water/solid interface, and in the theoretical understanding of the processes at the molecular scale, only a limited number of models is available to-date to describe the different phenomena and give reliable estimates of the $\zeta$-potential, the key parameter used to characterize the intensity of the EDL (Ishido and Mizutani 1981; Pride and Morgan 1991). In this study we use the model of Revil et al. (1999a), because it has been satisfactorily applied to study the electrokinetic processes in numerous studies (e.g., Revil et al. 1999b; Revil and Leroy 2001; Revil and Pessel 2002), and model predictions have often been found in good agreement with the measurements.

While the general formulation of the model is valid in the full range of $\mathrm{pH}$, an analytical solution is only available when $\mathrm{pH}$ is within the nearly neutral interval $(6<\mathrm{pH}<8)$. Since in most of the natural situations the pore fluid $\mathrm{pH}$ remains within these values (see for example Appelo and Postma 2005), in this study, we only consider the nearly neutral range. Furthermore, we restrict our model to situations where the $\zeta$-potential is smaller than $-20 \mathrm{mV}$, because for larger values the electrokinetic coupling is certainly negligible and therefore not of interest. In this subsection we only summarize the main equations of the theory, as implemented in our numerical model. Details of the theoretical development and discussion can be found in Revil et al. (1999a).

According to Revil et al. (1999a), the $\zeta$-potential can be closely approximated with the electrical potential of the Stern-layer:

$$
\zeta \approx \varphi_{\mathrm{d}}=\frac{2 k_{\mathrm{B}} T}{3 e} \ln \left[\frac{\sqrt{8 \cdot 10^{3} \varepsilon_{o} \varepsilon_{r} k_{\mathrm{B}} T N_{a}}}{2 e \Gamma_{s}^{0} K_{\mathrm{m}}}\left(\frac{C_{a}+C_{f}+10^{-p H}}{\sqrt{I_{c}}}\right)\left(10^{-p H}+C_{f} K_{\mathrm{Me}}\right)\right]
$$

where $C_{f}$ is salinity, $C_{a}$ is the concentration of acid in free electrolyte, and $I_{c}$ the ionic strength of the solution.

The chemical-physical constants used for the model (e.g., equilibrium dissociation constants for the surface groups) have also been taken from the literature (Revil et al. 1999a; Bockris and Reddy 1998) and are listed in the List of Symbols table. Electrical conductivity of the pore fluid is instead computed as (e.g., Bockris and Reddy 1998):

$$
\sigma_{f}=\sum_{i=1}^{\mathrm{ns}} z_{i} F C_{i} u_{i}^{\mathrm{conv}}
$$

The dielectric constant of water shows only a weak dependence on the ionic concentration, and we assume it constant (Bockris and Reddy 1998). Temperature has instead a potentially large influence, affecting (i) the electrical conductivity of the solution, (ii) the surface electrical conductance and (iii) the $\zeta$-potential. A linear dependence of all these quantities has been found to provide reasonable agreement with the measurements (e.g., Revil et al. 1999b), and therefore we account for temperature changes using the following equations: 


$$
\begin{aligned}
\sigma_{f}(T) & =\sigma_{f}\left(T_{o}\right)\left[1+\alpha_{f}\left(T-T_{o}\right)\right] \\
\Sigma_{s}(T) & =\Sigma_{s}\left(T_{o}\right)\left[1+\alpha_{s}\left(T-T_{o}\right)\right] \\
\zeta(T) & =\zeta\left(T_{o}\right)\left[1+\alpha_{\zeta}\left(T-T_{o}\right)\right]
\end{aligned}
$$

where $T_{0}$ and $T$ are the reference $(298 \mathrm{~K})$ and actual temperatures, respectively. The $\alpha$ 's correction coefficients are also taken from the literature (Revil et al. 1999b) and are listed in the List of Symbols table.

\subsection{Model Implementation}

A pore-network approach is used to represent the inner geometry of the porous medium. The network is composed of cylindrical interconnected capillaries. The electrical potential and fluid pressure are computed at the nodes of the network using a discretized form of Eqs. 8 and 9 with mass and charge conservation (see Bernabé 1998; Li and Kwok 2003).

Constant fluid pressure is set on two opposite boundaries of the pore-network, while the remainder boundaries are impermeable (to fluid flow) and insulating (to electrical current). The resulting system of linear equations is symmetric and has $2 n$ degrees of freedom. We tested both a preconditioned iterative conjugate gradient and a Cholesky decomposition to solve the system of equations. We observed that the iterative solver becomes unstable when the capillary radius distribution covers more than one order of magnitude due to poor conditioning of the system matrix. For this reason, in all the simulations we used the direct Cholesky decomposition, as implemented in the LAPACK library (Anderson et al. 1999). The main drawback of this approach is that only relatively small pore-networks can be considered. Nevertheless, as discussed in the next section, the size of the pore-network we use still allows drawing statistically significant conclusions.

Once the fluid pressure and the electrical potential at each node of the network are computed, the bulk intrinsic permeability is computed from the total volumetric flow using Darcy's equation.

\section{Numerical Experiments}

\subsection{Sensitivity Analysis on a Single Capillary}

The model we developed was initially used to assess the relative importance of each chemical-physical parameter of the porous medium, and to evaluate the extent of permeability reduction expected as a function of the pore (i.e., capillary) radius. In the following, simulation results are presented in terms of relative permeability, computed as the ratio between the permeability without and with the electrokinetic coupling. This is equivalent to the ratio between the intrinsic permeability measured with an inert gas and that measured using water in laboratory experiments.

We defined a base case condition, with geometrical and chemical physical properties based on realistic values for a fine-grained porous medium (e.g., a shale or clayey material). The numerical values of the main parameters are reported in Table 1.

First, we studied the permeability reduction for capillaries of different sizes. We found that, for the chemical-physical conditions considered in the simulation, the difference between the intrinsic permeability with and without electrokinetic coupling is small and only when the pore-radius is smaller than $0.5 \times 10^{-6} \mathrm{~m}$ the electrokinetic coupling becomes noticeable 
Table 1 Base case definition for the sensitivity analysis

\begin{tabular}{lll}
\hline Property & Value & Units \\
\hline Capillary radius $a$ & $10^{-6}$ & $\mathrm{~m}$ \\
Temperature $T$ & 293 & $\mathrm{~K}$ \\
Ion mobility $\mu_{i}$ & $4.3 \times 10^{-8}$ & $\mathrm{~m}^{2} \mathrm{~V}^{-1} \mathrm{~s}^{-1}$ \\
Surface charge density $\Gamma_{s}^{0}$ & 10 & sites nm \\
pH & 6.5 & - \\
Dielectric constant, water $\varepsilon_{r}$ & 80.0 & - \\
\hline
\end{tabular}

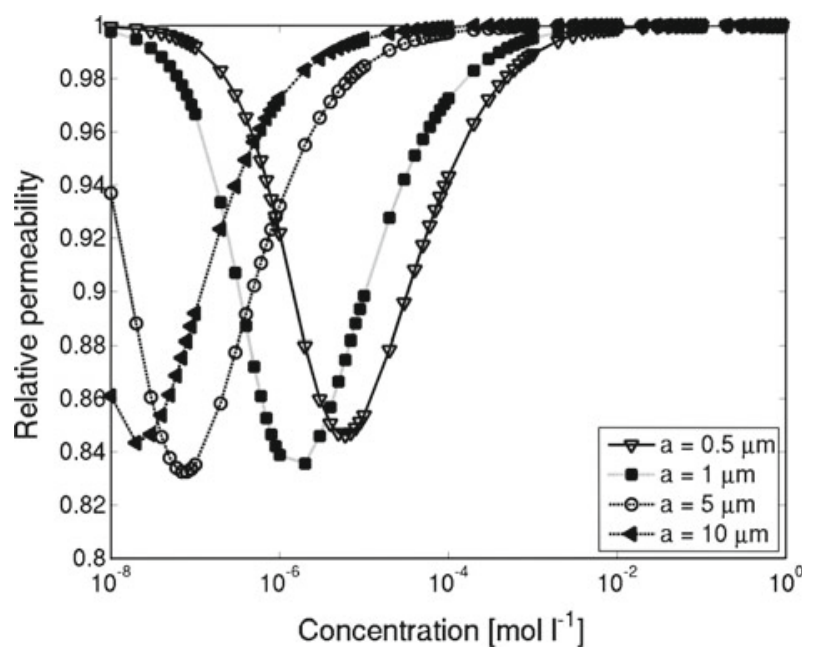

Fig. 2 Sensitivity analysis on a single capillary. Reduction of the intrinsic permeability as a function of the pore-fluid concentration for capillaries of different sizes. Note that concentrations smaller than $510^{-3} \mathrm{~mol}^{-1}$ are not realistic in natural conditions

(Fig. 2). We also observed that the extent of permeability reduction is a function of the ion concentration in the pore fluid. An inverse correlation between the two quantities exists, because the Debye length (i.e., the thickness of the EDL) decreases as the ionic strength of the solution increases. This in turn reduces the efficiency of the electrokinetic coupling, as quantified by the dimensionless electrokinetic radius $\kappa a$. This is a first important conclusion. According to our simulations, in porous media the effect of electrokinetic coupling is always limited to materials with effective pore-radius smaller than $0.5 \mu \mathrm{m}$. In practice, however, this value is further reduced because the salinity of the pore-fluid is always larger than $5 \times 10^{-3} \mathrm{~mol}^{-1}$. For example, aquifers in contact with hydrocarbon reservoirs are in most cases formed by brackish to saline waters (see Table 2 for a definition and classification). Therefore, from our modeling results it is evident that electrokinetic coupling can not be responsible for the observed discrepancies in gas and water intrinsic permeabilities in hydrocarbon-bearing sandstones.

However, the theory predicts that several chemical-physical properties of the porous medium can affect the extent of permeability reduction. We therefore examined the sensitivity to some of these properties, starting from the surface charge density, $\Gamma_{\mathrm{s}}^{0}$. This parameter influences the $\zeta$-potential and, consequently, the cross-coupling coefficients $L_{i j}$. Under natural conditions, the surface charge density can vary from 4 to 5 sites $\mathrm{nm}^{-2}$ in clean silica 
Table 2 Salinity classes, ionic concentrations and corresponding electrical conductivity values

\begin{tabular}{llllll}
\hline & Distilled water & Fresh water & Brackish water & Saline water & Brine \\
\hline $\begin{array}{l}\text { Concentration } \\
(\text { ppm o mg 1 }\end{array}$ & $<0.1$ & $<500$ & $500-30,000$ & $30,000-50,000$ & $>50000$ \\
$\begin{array}{l}\text { Concentration } \\
\left(\text { mol 1 }^{-1}\right)\end{array}$ & $<1.5 \cdot 10^{-6}$ & $<8.0 \cdot 10^{-3}$ & $8.0 \cdot 10^{-3}-0.5$ & $0.5-0.8$ & $>0.8$ \\
$\begin{array}{l}\text { Electrical conductivity } \\
\left(\mathrm{S} \mathrm{cm}^{-1}\right)\end{array}$ & $<2 \cdot 10^{-5}$ & $<1 \cdot 10^{-3}$ & $1 \cdot 10^{-3}-6 \cdot 10^{-2}$ & $6 \cdot 10^{-2}-1 \cdot 10^{-1}>1 \cdot 10^{-1}$ \\
\hline
\end{tabular}

From Appelo and Postma (2005)

sands (Revil et al. 1999a; Revil et al. 1999b) and increases with the increase in clay and oxide content. The value can be estimated from the cation exchange capacity of the formation, CEC [meq $\mathrm{g}^{-1}$ ] (Waxman and Smits 1968):

$$
\Gamma_{\mathrm{s}}^{0}=e \rho_{\mathrm{g}}\left(\frac{1-n}{n}\right) \varphi_{\mathrm{w}} C E C
$$

Using this formula and tabulated values of $C E C$ (for example, see Waxman and Smits 1968) it is possible to estimate that the largest possible surface charge density $\Gamma_{\mathrm{s}}^{0}$ is of the order $80-100$ sites $\mathrm{nm}^{-2}$. It should, however, be kept in mind that this value is referred to a unit of the inner surface area of the porous medium. This geometrical property is certainly not correctly reproduced in the pore-network representation we adopted, because idealizing pores as cylindrical capillaries strongly underestimate their surface area. Therefore, a larger value could be used in the simulations.

Results for this suite of simulations are shown in Fig. 3. The changes in permeability over the range of surface density considered are relatively small. Quite surprisingly, however, the variation is non monotonic. Relative permeability first decreases in the range $4-20 \mathrm{~nm}^{-1}$, while for larger values shows a weak but steady increase. This is due to two competing mechanisms: (i) the increase of the $\zeta$-potential, which in turn augments the electrokinetic coupling and leads to a smaller intrinsic permeability, and (ii) the increase in surface conductance, which results in a weaker electrokinetic coupling, because locally the electrical charges can be balanced more easily. This behavior is, however, only visible when the ionic strength of the solution is small. For medium and high concentrations, the permeability change is almost negligible, at least for the range of pore-radii considered. The pore-solution $\mathrm{pH}$ has a similar effect on the $\zeta$-potential (results not shown). Recall that, as discussed in Sect. 2 above, we only evaluated the $\mathrm{pH}$ range of $6-8$ because the EDL model is not correct outside this interval. Indeed, the $\mathrm{pH}$ in natural conditions mainly belongs to this interval, even in soils where the $\mathrm{pH}$ can be slightly acidic (about 6). We observed that the effect of $\mathrm{pH}$ is larger in pore fluids with lower salinity. This is expected, because the ion concentration change induced by the $\mathrm{pH}$ variation (increase or decrease of $\mathrm{H}^{+}$in solution) would be more similar to the background ionic concentration (recall $\left[H^{+}\right] \sim \log _{10}(p H)$ ). Again, however, for realistic salinities of the pore-fluid the sensitivity to $\mathrm{pH}$ is almost negligible. This can be explained considering that the salt concentration is much larger than the proton concentration, and therefore completely hides the changes in solution composition resulting from $\mathrm{pH}$ variations.

The physical property with larger impact we found is temperature, as illustrated in Fig. 3 (left panel). Temperature influences the three key mechanisms for the electrokinetic coupling, namely the electrical conductivity of water, the surface conductance, and the $\zeta$-potential (Eqs. 18-20). The variation of permeability reduction is nonlinear, with the permeability 

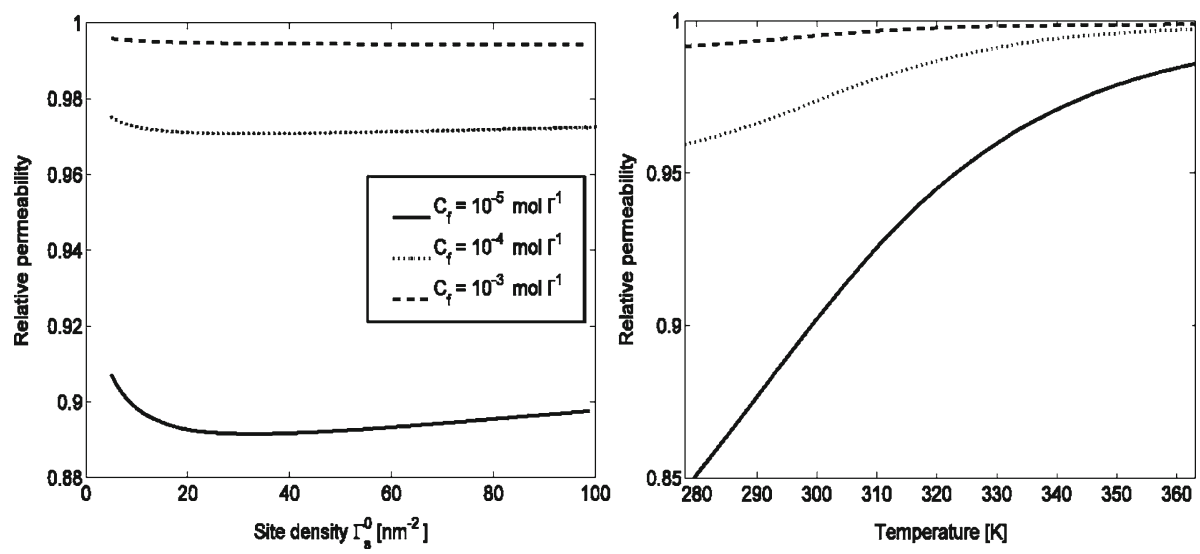

Fig. 3 Effect of charged site density and temperature on permeability reduction. Three pore solutions with different ionic concentrations are considered, while the pore-size is fixed $\left(10^{-6} \mathrm{~m}\right)$. Simulation results illustrate that permeability reduction is only slightly sensitive to both variables

steadily increasing with temperature. At about $60^{\circ} \mathrm{C}$ water and gas (i.e., with and without electrokinetic effects) permeabilities show almost no difference, even at very small ionic concentrations (nearly for distilled water). Since earth temperature increases with depth, this is further evidence that the electrokinetic coupling is not a likely mechanism that explains the observed differences in gas/fluid permeability in deep hydrocarbon reservoirs.

Indeed, the decrease in electrokinetic coupling with temperature is mainly due to the increased ion mobility at higher temperatures. The determination of the electrophoretic ion mobility (i.e., the mobility of ions under a unit external electric field) has large uncertainties, but both experiments and theory show that the mobility of ions in the presence of an EDL is strongly reduced compared to the values in clean fresh water, and the difference can be as large as two orders of magnitude (e.g., Rotenberg et al. 2006, 2007). Therefore, we investigated how the electrokinetic coupling and the resulting permeability variation change when the ionic mobility is reduced. Results are shown in Fig. 4. A strong, nonlinear permeability reduction is observed for the solution with weak ionic strength. For realistic solutions, however, the permeability change is almost negligible. Once again, this is because of the small thickness of the EDL compared to the size of the capillary. Therefore, the effect of reduced ionic mobility becomes important when the size of the capillary is comparable to the inverse of the Debye length.

\subsection{Pore-Network Simulations}

The sensitivity analysis we illustrated in the previous sub-section clearly shows that electrokinetic coupling in shaly sandstones does not explain in practice the different permeability measured both using an inert gas and a fluid water solution. Indeed, our single-capillary simulation results show that electrokinetic coupling possibly affects porous material with small average pore-radii. This is the case, for example, of clays and cement pastes. However, in order to elucidate the sensitivity of electrokinetic coupling and permeability decrease to more realistic pore-size distributions and more complex geometries, we also conducted a suite of pore-network simulations. 


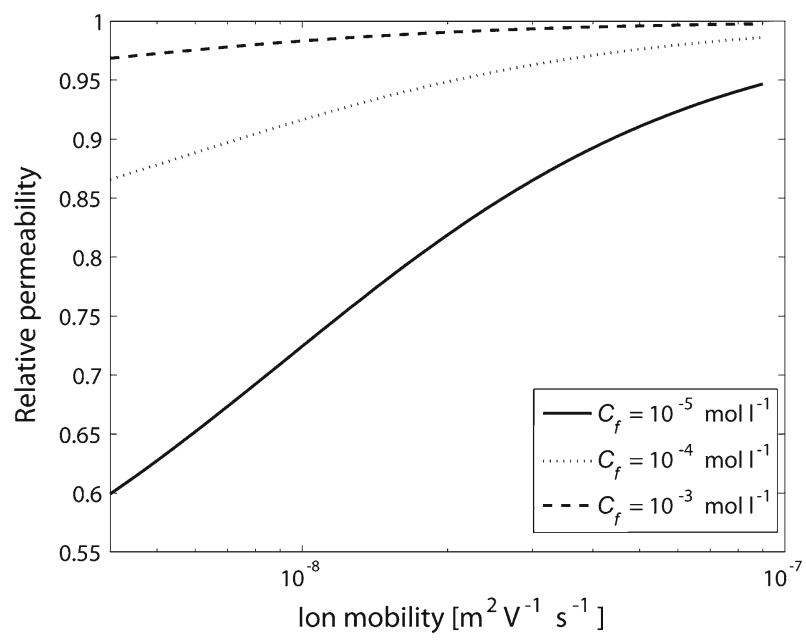

Fig. 4 Dependence of permeability reduction on ion mobility. Three water solutions with different ionic concentrations are considered. The capillary radius is fixed $\left(10^{-6} \mathrm{~m}\right)$, but similar results are obtained using different pore-radii, with the extent of permeability reduction increasing as the size of the capillary decreases

\subsubsection{Description of the Pore-Network}

Pore-network simulations have often been used to investigate transport properties of porous media, considering both single and multi-phase conditions. To this end, several network geometries and pore configurations have been proposed and used to assess the relative importance of the porous medium's micro-geometrical properties, such as porosity, poreconnectivity, and tortuosity (see for example Dullien 1992; Rajaram 1997; Blunt 1998; Peat et al. 2000; Vogel 2000). In this study, we are solely interested in the pore-size distribution, because the extent of electrokinetic coupling is only affected by the size of the EDL relative to the pore-radius. Therefore, based on Bernabé 1998, we use a simple quadrilateral network, with constant node spacing and connectivity (each node is connected to its 4 nearest neighbors). We use two-dimensional networks, composed of $50 \times 50$ nodes, resulting in a system of linear equations with 5,000 degrees of freedom. Based on experimental observations, we assume that the pore-size distribution follows a truncated log-normal distribution (Dullien 1992). While the pore-networks we consider here are certainly simplistic and do not represent realistic porous media in terms of pore-shapes and connectivity, the only geometrical parameter that affects the extent of electrokinetic coupling is the pore-radius. Therefore, all the other micro-geometrical properties influence in the same way the permeability with and without electrokinetic effects, and, consequently, our conclusions will not be affected by the simplifying assumption that we make regarding the network topology and pore-shapes.

\subsubsection{Methodology}

We tested six different pore-size distributions. The mean was varied from $7.5 \times 10^{-7} \mathrm{~m}$ to $5.0 \times 10^{-8} \mathrm{~m}$, while the standard deviation was fixed for each case to $20 \%$ of the mean (note that these values do not refer directly to the log-normal distribution, but instead to the corresponding Gaussian). Table 3 reports the mean and standard deviation of each pore-size 
Table 3 Pore-size distributions considered for the network simulations

The standard deviation is computed as a percent of the mean radius. Reported are the parameters of the corresponding normal distribution

\begin{tabular}{llll}
\hline Case \# & Mean radius $(\mathrm{m})$ & Standard deviation $(\mathrm{m})$ & $r_{25}(\mathrm{~m})$ \\
\hline 1 & $7.5 \times 10^{-7}$ & $1.5 \times 10^{-7}$ & $6.0 \times 10^{-7}$ \\
2 & $5.0 \times 10^{-7}$ & $1.0 \times 10^{-7}$ & $4.0 \times 10^{-7}$ \\
3 & $2.5 \times 10^{-7}$ & $5.0 \times 10^{-8}$ & $2.0 \times 10^{-7}$ \\
4 & $1.0 \times 10^{-7}$ & $2.0 \times 10^{-8}$ & $8.0 \times 10^{-8}$ \\
5 & $7.5 \times 10^{-8}$ & $1.5 \times 10^{-8}$ & $6.0 \times 10^{-8}$ \\
6 & $5.0 \times 10^{-8}$ & $1.0 \times 10^{-8}$ & $4.0 \times 10^{-8}$ \\
\hline
\end{tabular}

distributions, together with the $r_{25}$ : this parameter indicates the lower 0.25 percentile, meaning that, on average, the radius of $25 \%$ of the capillaries of each pore-network is smaller than the $r_{25}$. This value is a useful compact indicator of the pore-size distribution, and we will use it in the next sub-section to present the results of our pore-network simulations.

In order to identify the expected permeability reduction for each of the studied pore-size distributions, we employed a stochastic methodology. For each of the six cases, multiple realizations of the pore-network were generated. For each realization we computed the intrinsic permeability both with and without electrokinetic coupling. Finally, the relative permeability (i.e., the ratio between the two computed values) for each network and its mean value were calculated.

Numerical experiments were conducted to identify the number of realizations required to give stable, statistically significant results. To this end, we generated 1,000 realizations, and we computed the intrinsic permeability without electrokinetic coupling. Next we calculated, for each additional realization, the resulting mean and standard deviation. The relative error is computed as $E=1-v_{i} / v_{\infty}, v_{i}$ is the value (mean or standard deviation), considering the first $i$ realizations, and $v_{\infty}$ is the corresponding value resulting from averaging the mean or standard deviation for the last 50 realizations. This procedure guarantees that all the possible values of intrinsic permeability are accounted for when computing the average value of the permeability reduction.

We arbitrarily assumed that the Monte Carlo method converges when the residual error for both the mean and the standard deviation is smaller than $5 \%$. In order to achieve this accuracy, at least 450 realizations are required, and, therefore, in all the following simulations for each pore-size distribution, we generated 500 pore-networks for each set of parameters.

From the sensitivity analysis described in Sect. 3.1, we concluded that ion mobility is the electrochemical parameter with the largest impact on the extent of permeability reduction. Experiments and numerical simulations have found that the electrophoretic mobility of ions in clays is reduced compared with free-water conditions. The reduction can be as large as one order of magnitude, with most of the experiments showing an average electrochemical mobility between $1 \times 10^{-8}$ and $2 \times 10^{-8} \mathrm{~m}^{2} \mathrm{~V}^{-1} \mathrm{~s}^{-1}$. In order to better understand the behavior of real porous media, we repeated the Monte Carlo pore-network simulations using three values for the electrophoretic mobility: (i) the free-water mobility, assumed equal to $5 \times 10^{-8} \mathrm{~m}^{2} \mathrm{~V}^{-1} \mathrm{~s}^{-1}$ (e.g., the mobility of $\mathrm{Na}$ is $4.67 \times 10^{-8} \mathrm{~m}^{2} \mathrm{~V}^{-1} \mathrm{~s}^{-1}$, Bockris and Reddy (1998); (ii) the average electrophoretic ionic mobility measured in clays, $1 \times 10^{-8} \mathrm{~m}^{2} \mathrm{~V}^{-1} \mathrm{~s}^{-1}$; and (iii) $5 \times 10^{-9} \mathrm{~m}^{2} \mathrm{~V}^{-1} \mathrm{~s}^{-1}$, corresponding to the free-water mobility reduced by one order of magnitude. This latter value may be slightly too small, and, therefore, the resulting permeability should be viewed more as a lower bound. 
In summary, we ran 18 sets of Monte Carlo simulations with each set composed of 500 pore-networks and taking about $30 \mathrm{~h}$ of CPU time on a modern $3.2 \mathrm{GHz}$ machine.

\subsubsection{Simulation Results}

An overview of simulation results is shown in Fig. 5. Each curve is relevant to the results obtained using a different ionic mobility. The top and bottom curves represent the approximate upper and lower bounds for the possible range of permeability variations, because the corresponding ionic mobilities bracket the realistic range of ionic mobilities in clays and shaly porous media. We represent each of the six studied pore-radius distributions with the value assumed by the $r_{25}$.

From Fig. 5, we deduct that ionic mobility has a very strong impact on the amount of permeability reduction in the presence of electrokinetic coupling. The range of possible variation for the permeability reduction is relatively large. When the ionic mobility is set to free-water mobility, the variation is always smaller than $5 \%$ of the permeability without electrokinetic coupling, and less than $2 \%$ when the characteristic radius $r_{25}$ is larger than $0.1 \mu \mathrm{m}$. On the other hand, when the ion mobility is set to its lower bound, the relative permeability can be as low as 0.7 , and lies between 0.85 and 0.95 when $r_{25}>0.1 \mu \mathrm{m}$. The more realistic estimate is, however, given by the middle line, because the ionic mobility is set to a more realistic and measured value. Therefore, we can conclude that, from our numerical experiments, it is reasonable to estimate an average $5 \%$ reduction in permeability when the characteristic pore-radius is between 1 and $5 \times 10^{-7} \mathrm{~m}(0.1$ to $0.5 \mu \mathrm{m})$, and a reduction of $10-15 \%$ when

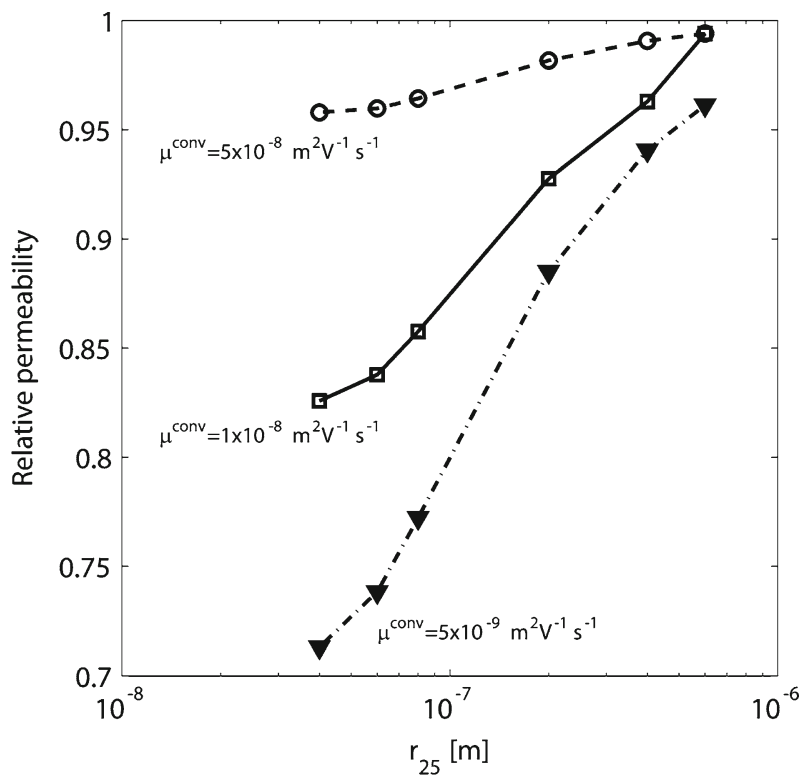

Fig. 5 Average permeability reduction resulting from the pore-network simulations. The top line (open circles) is relevant to modeling results using typical free-water mobility, while the bottom line (closed triangles) is a possible lower bound for ion mobility in clays. The middle line (open squares) shows the modeling results obtained using an average ion mobility measured in clays. From simulation results we concluded that permeability reduction due to electrokinetic coupling can never be larger than $20 \%$ of the permeability without electrokinetic effects 
$r_{25}$ is around $5 \times 10^{-8} \mathrm{~m}$. Clearly, this latter value is extremely small, and only very few geological materials (such as compacted clays) belong to this range. Furthermore, it must be considered that our model is likely to overpredict the permeability reduction for two main reasons: First, the EDL model underestimates the surface conductivity in clays, since it does not take into account all the possible contributions (for example, $\mathrm{Al}$ and $\mathrm{Fe}$ oxides are not considered); Second, the pore-network simulations were set-up considering a water phase with small ionic strength, e.g., ion concentrations typical of freshwater. As clearly shown by the sensitivity analysis above, high ionic strength would result in a reduced electrokinetic coupling (owing to the reduced size of the EDL), and, therefore, in a smaller permeability reduction.

\section{Summary and Conclusions}

In this study, we have conducted a detailed, process-based investigation of one of the mechanisms listed by several previous researches as potentially responsible for some reduction in the intrinsic water permeability compared to the permeability measured using an inert gas, for example, nitrogen. Our simulations clearly show that electrokinetic coupling does not significantly influence the water flow when the effective pore-radius is larger than $5 \times 10^{-7} \mathrm{~m}$, and the composition of the pore-solution is realistic (ion concentration or salinity larger than $10^{-3} \mathrm{~mol} \mathrm{l}^{-1}$ ). Therefore, we conclude that electrokinetic coupling is not significant in most of the hydrocarbon-bearing formations, for example, in sandstones and shaly sandstones. In these materials, other mechanisms (possibly the mobilization of fines and partial desaturation of the sample) must be called into play. Further to this, the sensitivity analysis we conducted showed that the electrophoretic mobility is the key parameter governing the extent of permeability reduction. However, the uncertainties about the value assumed by this electrochemical parameter in clays are large, and measurements are severely affected by errors.

We also studied the permeability reduction in more realistic porous geometries than a single capillary. Pore-network simulations of fine-grained materials (mean pore-radius smaller than $1 \times 10^{-6} \mathrm{~m}$ ), using a log-normal pore-radius distribution were conducted, which further confirmed our previous findings. We observed that, if the pore-size distribution has a large fraction of pores larger than $5 \times 10^{-7} \mathrm{~m}$, then the effect of electrokinetic coupling on the permeability is very limited, and the permeability reduction never exceeds $2 \%$. On the other hand, when the pores are smaller than this value, electrokinetic coupling becomes potentially important. The amount of permeability reduction is strongly sensitive to the ion mobility, but never exceeds $15-20 \%$, and this value is possibly overestimated. Experiments have, however, shown that in numerous situations the intrinsic permeability measured using an inert gas is several times larger than the permeability measured with water. Our pore-scale simulations clearly show that electrokinetic coupling is, at most, responsible for a small part of the observed reduction. On the other hand, our conclusions also show that for critical applications, such as the design of liners for toxic landfills or radionuclides repositories, measurements of the intrinsic permeability must be conducted using both water and gas.

In this study, we only investigated one of the possible mechanisms responsible for the differences in intrinsic permeabilities. Most of the investigators suggest that the measured difference is mainly due to the sample desaturation and clogging of pores due to migration of fines. In our view, however, these processes do not fully agree with the measurements. For example, some of the experiments seem to suggest that the mechanism is somehow reversible (i.e., the permeability with gas is the same before and after measuring the permeability with water) and, therefore, is not compatible with pore-clogging. Future research efforts should 
focus on addressing these questions and provide a fundamental, mechanistic understanding of the different processes taking place.

Acknowledgements This research was made possible by partial funding from the EU FP7 collaborative project iSOIL "Interactions between soil related sciences-Linking geophysics, soil science and digital soil mapping". We also wish to acknowledge the support received from Eni E\&P, Milan, Italy. The useful discussions we had with Luigi Terzi and Stefano Mantica are also gratefully acknowledged.

\section{References}

Anderson, E., Bai, Z., Bischof, C., Blackford, S., Demmel, J., Dongarra, J., Du Croz, J., Greenbaum, A., Hammarling, S., McKenney, A., Sorensen, D.: LAPACK Users' Guide. 3rd edn. Society for Industrial and Applied Mathematics, Philadelphia, PA (1999)

Appelo, C.A.J., Postma, D.: Geochemistry, groundwater and pollution. 2nd edn. A.A. Balkema Publishers, Leiden, the Nederlands (2005)

Baraka-Lokmane, S.: Hydraulic versus pneumatic measurements of fractured sandstone permeability. J. Petroleum Sci. Eng. 6, 183-192 (2002)

Bear, J.: Dynamics of Fluids in Porous Media. Dover Publications, NY, USA (1988)

Bernabé, Y.: Streaming potentials in heterogeneous networks. J. Geophys. Res. 103(B9), 20827-20841 (1998)

Blunt, M.J.: Physically based network modeling of multiphase flow in intermediate-wet Media. J. Petroleum Sci. Eng. 20(3-4), 117-125 (1998)

Bockris, J.O’M., Reddy, A.K.N.: Modern electrochemistry, vol. 1: Ionics. 2nd edn. Plenum press, New York and London (1998)

Bowen, W.R., Jenner, F.: Electroviscous effects in charge capillaries. J. Colloid Interface Sci. 173, 388-395 (1995)

Capuani, F., Pagonabarraga, I., Frenkel, D.: Discrete solution of the electrokinetic equations. J. Chem. Phys. 121(2), 973-986 (2004)

Capuani, F., Pagonabarraga, I., Frenkel, D.: Mesoscopic lattice modeling of electrokinetic phenomena. Comput. Phys. Commun. 169, 192-196 (2005)

Dana, E., Skoczylas, F.: Gas relative permeability and pore structure of sandstones. Int. J. Rock Mech. Min. Sci. 36, 613-625 (1999)

Dullien, F.A.L.: Porous media: fluid transport and pore-structure. 2nd edn. Academic Press, San Diego (1992)

Guéguen, Y., Palciauskas, V.: Introduction to the physics of rocks. Princeton University Press, Princeton, NJ, USA (1994)

Hildreth, D.: Electrokinetic flow in fine capillary channels. J. Phys. Chem. 74(9), 2006-2015 (1970)

Hlushkou, D., Apanasovich, V., Seidel-Morgenstern, A., Tallarek, U.: Numerical simulations of electrokinetic microfluidics in colloidal systems. Chem. Eng. Commun. 193, 826-839 (2006)

Ishido, T., Mizutani, H.: Expermental and theoretical basis of electrokinetic phenomena in water-rock systems and its application to geophysics. J. Geophys. Res. 86, 1763-1775 (1981)

Jones, F.O., Owens, W.W.: A laboratory study of low-permeability gas sands. J. Petroleum Tech. SPE paper 7551, pp. 1631-1640 (1980)

Juhàsz, I.: The central role of Qv and formation water salinity in the evaluation of Shaly formations. In: Proceedings of the SPWLA 20th Annual Logging Symposium, Tulsa (1979)

Keijzer, ThJ.S., Kleingeld, P.J., Loch, J.P.G.: Chemical osmosis in compacted clayey material and the prediction of water transport. Eng. Geol. 53, 151-159 (1999)

Li, B., Kwok, Y.: Lattice Boltzmann model of microfluidics in the presence of external forces. J. Colloid Interface Sci. 263, 144-151 (2003)

Lyklema, J.: Surface conduction. J. Phys. Condens. Matter 13, 5027-5034 (2001)

Onsager, L.: Reciprocal relations in irreversible processes. II. Phys. Rev. 38, 2265-2279 (1931)

Peat, D.M.W., Matthews, G.P., Worsfold, P.J., Jarvis, S.C.: Simulation of water retention and hydraulic conductivity in soil using a three-dimensional network. Eur. J. Soil Sci. 51, 65-79 (2000)

Press, W.H., Flannery, B.P., Teukolsky, S.A., Vetterling, W.T.: Numerical Recipes in FORTRAN: the Art of Scientific Computing, 735 pp. Cambridge University Press, New York (1998)

Pride, S., Morgan, F.D.: Electrokinetic dissipation induced by seismic waves. Geophysics 56, 914-925 (1991)

Raffenspergen, J.P., Ferrel, R.E. Jr.: Empirical model of intrinsic permeability in reactive clay-bearing sands. Water Resour. Res. 27(11), 2835-2844 (1991)

Rajaram, H.: Prediction of relative permeabilities for unconsolidated soils using pore-scale network models. Water Resour. Res. 33(1), 43-52 (1997)

Revil, A., Leroy, P.: Hydroelectric coupling in clayey material. Geophys. Res. Lett. 28(8), 1643-1646 (2001) 
Revil, A., Pessel, M.: Electroosmotic flow and the validity of the classical Darcy equation in silty shales. Geophys. Res. Lett. 29(9), 1300 (2002)

Revil, A., Pezard, P.A., Glover, P.W.J.: Streaming potential in porous media 1. Theory of the zeta potential. J. Geophys. Res. 104(B9), 20021-20031 (1999)

Revil, A., Schwaeger, H., Cathles, L.M. III., Manhardt, P.D.: Streaming potential in porous media 2. Theory and application to geothermal systems. J. Geophys. Res 104(B9), 20033-20048 (1999)

Rotenberg, B., Dufreche, J.F., Bagchi, B., Giffaut, E., Hansen, J.P., Turq, P.: Ion dynamic in compacted clays: Derivation of a two-state diffusion reaction scheme from the lattice Fokker-Plank equation. J. Chem. Phys. 123, 154701 (2006)

Rotenberg, B., Marry, V., Dufreche, J.F., Giffaut, E., Turq, P.: A multiscale approach to ion diffusion in clays: Building a two-state diffusion-reaction scheme from microscopic dynamics. J. Colloid Interface Sci. 309, 289-295 (2007)

Soma, J., Papadopulos, K.D.: Flow on dilute, sub-micron emulsions in granular porous media: effect of $\mathrm{pH}$ and ionic strength. Colloids Surf. A Physicochem. Eng. Asp. 101, 51-61 (1995)

Swanson, B.F.: A simple correlation between air permeabilities and stressed brine permeabilities with mercury capillary pressures. Presented at 53rd Annual Technical Conference and Exhibition of SPE, Houston, TX, SPE paper 8234 (1978)

Swanson, B.F.: A simple correlation between permeabilities and mercury capillary pressures. J. Petroleum Tech. 2498-2504 (1981)

Vogel, H.J.: A numerical experiment on pore-size, pore connectivity, water retention, permeability and solute transport using network models. Eur. J. Soil Sci. 51, 99-105 (2000)

Waxman, M.H., Smits, L.H.: Electrical conductivities in oil-bearing shaly sands, SPE J. 8, 107-122 (1968)

Wilson, M.D.: Origins of clays controlling permeability in tight gas sands. J. Petrol. Tech., SPE paper 9843, pp. 2871-2876 (1982)

Xuan, X., Li, D.: Analysis of electrokinetic flow in microfluidic networks. J. Micromech. Microeng. 14, 290-298 (2004) 